\title{
O Índice de Memória Operacional do WISC-IV na Avaliação do TDAH
}

\author{
Carolina da Silva Büttow ${ }^{1}$ \\ Vera Lúcia Marques de Figueiredo ${ }^{1}$ \\ ${ }^{1}$ Universidade Católica de Pelotas, Pelotas, RS
}

\begin{abstract}
Resumo
O objetivo do presente estudo foi analisar a sensibilidade do Índice de Memória Operacional (IMO) da Escala Wechsler de Inteligência para Crianças - $4^{a}$ Edição, na identificação de dificuldades em crianças e adolescentes com o diagnóstico de Transtorno de Déficit de Atenção e Hiperatividade (TDAH). Participaram 40 sujeitos, entre 06 e 16 anos, com diagnóstico neurológico prévio de TDAH. Foi utilizada a Escala de Atenção de Swanson, Nolan e Pelham - 4ª Edição (SNAP - IV) para pais/responsáveis e os subtestes do WISC-IV que formam o Índice de Memória Operacional (Dígitos, Aritmética e Sequência de Números e Letras). Entre os resultados, identificou-se IMO rebaixado quando comparado com o escore esperado para a população geral e, em Dígitos, observou-se o menor desempenho do grupo. Concluiu-se que o Índice de Memória Operacional é adequado para diagnosticar e avaliar déficits de atenção e de memória em crianças e adolescentes com TDAH.

Palavras-chave: TDAH, Escala de Inteligência Wechsler para Crianças, memória operacional, atenção
\end{abstract}

\section{The WISC-IV Operational Memory Index in the Assessment of ADHD}

\begin{abstract}
The objective of the present study was to analyze the sensitivity of the "Operational Memory Index" (OMI) of the Wechsler Intelligence Scale for Children- 4th Edition in the identification of difficulties in children and adolescents with the diagnosis of Attention Deficit Hyperactivity Disorder. Participants included forty subjects, between 06 and 16 years old, with a previous neurological diagnosis of ADHD. We used the Swanson Attention Scale, Nolan and Pelham - 4th Edition (SNAP-IV) Parent Rating Scale for parents/guardians and the WISC-IV subtests that form the Operational Memory Index (Digits, Arithmetic and Sequence of Numbers and Letters). Among the results, IMO was lower when compared to the expected score for the general population and in Digits there was a lower performance by the group. It was concluded that the Operational Memory Index is adequate to diagnose and evaluate attention and memory deficits in children and adolescents with ADHD.
\end{abstract}

Keywords: ADHD, wechsler intelligence scale for children, operational memory, attention

\section{Índice de Memoria Operativa del WISC-IV en la Evaluación del TDAH}

\begin{abstract}
Resumen
El objetivo del presente estudio fue analizar la sensibilidad del "Índice de Memoria Operativa" (IMO) de la Escala Wechsler de Inteligencia para Niños - $4^{a}$ Edición en la identificación de dificultades en niños y adolescentes con el diagnóstico de Trastorno de Déficit de Atención e Hiperactividad (TDAH). Participaron 40 sujetos, entre 06 y 16 años, con diagnóstico neurológico pre-

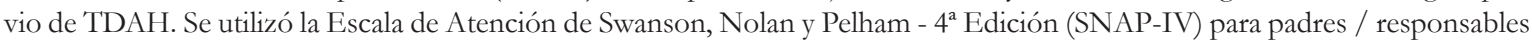
y los sub-test del WISC-IV que forman el Índice de Memoria Operativa (Dígitos, Aritmética y Secuencia de Números y Letras). Entre los resultados, se identificó el IMO, rebajado al ser comparado con el resultado esperado para la población general, y en Dígitos se observó el menor desempeño del grupo. Se concluyó que el Índice de Memoria Operativa es adecuado para diagnosticar y evaluar déficit de atención y de memoria en niños y adolescentes con TDAH.

Palabras-clave: TDAH, Escala de Inteligencia Wechsler para Niños, Memoria Operativa, Atención
\end{abstract}

O Transtorno de Déficit de Atenção e Hiperatividade (TDAH), segundo Effgem, Canal, Missawa e Rossetti (2017), é uma síndrome neurocomportamental com sintomas classificados em três categorias: desatenção, hiperatividade e impulsividade. Caracteriza-se por um nível inapropriado de atenção em relação ao esperado para a idade, com ou sem impulsividade e/ ou hiperatividade, levando a distúrbios motores, perceptivos, cognitivos e comportamentais que geram dificuldades globais no desenvolvimento infantil. Essa complexa desordem comportamental leva a criança a graus variados de comprometimento na vida social, emocional, escolar e familiar.

O Manual Diagnóstico e Estatístico de Transtornos Mentais da Associação Americana de Psiquiatria (DSM - V), em sua quinta edição (American Psychiatric Association [APA], 2014),classifica o TDAH em três tipos: com predomínio de desatenção, com predomínio de hiperatividade-impulsividade e com apresentação combinada. Esse manual apresenta uma lista de dezoito 
sintomas, sendo nove de desatenção, seis de hiperatividade e três de impulsividade (estes dois últimos computados conjuntamente), sendo que os indivíduos com até dezessete anos de idade precisam apresentar seis dos sintomas listados, enquanto indivíduos mais velhos precisam de apenas cinco. Os sintomas devem estar presentes antes dos doze anos de idade. Para serem considerados clinicamente significativos, devem estar presentes por pelo menos seis meses, serem nitidamente inconsistentes com a idade do indivíduo (ou seja, ser o indivíduo muito mais desatento ou inquieto do que o esperado para uma determinada idade) e haver comprometimento em pelo menos duas áreas diferentes (casa e escola, por exemplo) (APA, 2014).

Para Rohde e Mattos (2003), déficits de atenção significativos, associados ou não à hiperatividade, com frequência comprometem o rendimento escolar, já que a atenção seletiva a estímulos relevantes é condição necessária para a ocorrência das aprendizagens em geral e, em especial, as escolares. Para os autores, a criança com TDAH apresenta dificuldades para sustentar a atenção durante um tempo mais prolongado; a dificuldade também está presente ao selecionar a informação relevante em cada problema, de forma a estruturar e realizar uma tarefa. Essas dificuldades intensificam-se nas situações grupais, já que elas exigem atenção sustentada e seletiva para o manejo da grande quantidade de informação que é gerada.

Effgem et al. (2017), Guadagnini e Simão (2016) e Pereira, Leon, Dias e Seabra (2012) destacam que, além dos prejuízos escolares já citados, as crianças com TDAH podem apresentar também possíveis problemas cognitivos na atenção, nas funções executivas ou na memória. Os estudos, ao evidenciarem as relações entre indicadores de desatenção e hiperatividade e desempenho inferior em testes de funções executivas, passam a incluir as funções executivas nos modelos de explicação atuais de TDAH.

Messina e Tiedemann (2009) citam as funções executivas como responsáveis pela habilidade associada à memória de trabalho e funções correlatas, como planejamento, organização, atenção seletiva e controle inibitório. Estas são capacidades que permitem a um indivíduo perceber estímulos do seu ambiente, responder adequadamente, mudar de direção de modo flexível, antecipar objetivos futuros, considerar consequências e responder de modo integrado, utilizando todas as capacidades para alcançar um objetivo final.

A memória de trabalho ou memória operacional, como é denominada por Wechsler (2013, p. 04), “é a habilidade de manter-se consciente de uma informação recebida, desenvolver uma atividade, saber manipulá-la e, a partir dela, produzir um resultado". Caracteriza-se por ser um componente essencial para a organização perceptual e para outros processos cognitivos relevantes e, principalmente, caracteriza-se pelo fato de analisar a atenção, a concentração e a memória, assim como está intimamente relacionada ao aprendizado e às realizações. Em seus estudos, Alves, Neme e Cardia (2015) destacam que crianças com TDAH podem ter mais dificuldades que seus pares sem o transtorno em atividades que exijam a memória operacional.

Para o diagnóstico do TDAH, deve-se realizar uma avaliação neuropsicológica, aliada aos dados do exame clínico da criança (Alves, Neme, \& Cardia, 2015; Rohde \& Halpern, 2004; Valença \& Nardi, 2015). Os estudos de Coutinho, Mattos, Scmitz, Fortes e Borges (2009), Marcon, Sardagna e Schussler (2016) e Effgem et al. (2017) destacam ser necessário também a aplicação de escalas e questionários para pais e professores, pois nem sempre as crianças conseguem fazer um relato preciso acerca do seu comportamento.

Inseridas na avaliação neuropsicológica, as Escalas Wechsler de Inteligência para Crianças têm se mostrado como o instrumento capaz de fornecer um maior número de informações que podem auxiliar no diagnóstico de TDAH e no momento de traçar um plano de tratamento (Effgem, Canal, Missawa, \& Rossetti, 2017; Ignacio, Gonzales, Almeida, Andrade, \& Monteiro, 2008; Lopes, Farina, Welter, Esteves, \& Argimon, 2012; Rohde, Barbosa, Tramontina, \& Polanczyk, 2000; Schwean \& McCrimmon, 2008; Wechsler, 2013). Graeff e Vaz (2008) já apontavam que o fator de distratibilidade (Índice de Resistência à Distração - WISC-III), composto pelos subtestes de Dígitos e Aritmética, poderia auxiliar a hipótese diagnóstica de TDAH.

Lançada no Brasil em 2013, a $4^{a}$ versão da Escala Wechsler de Inteligência para Crianças (Weschler, 2013), trouxe algumas mudanças em relação à edição anterior (WISC-III), quanto ao conteúdo do instrumento, simplificando a estrutura fatorial, reformulando a constituição dos subtestes, incluindo novas provas e redefinindo os construtos subjacentes. $\mathrm{O}$ tradicional "Índice Fatorial Resistência à Distração", da versão anterior, recebeu a denominação de Working Memory, que já era utilizado pelo WAIS - III (Vidal, Figueiredo, \& Nascimento, 2011). O fator que envolve a avaliação da atenção denominou-se Índice de Memória Operacional (IMO), constituído pelos subtestes Dígitos 
e Sequência de Números e Letras - tendo o subteste Aritmética como suplementar.

Para aferir com precisão a memória, o subteste Sequência de Números e Letras, do WAIS - III, foi adaptado e incorporado ao WISC-IV. O subteste Aritmética também sofreu modificações para que os conhecimentos matemáticos requeridos fossem mais apropriados para as diferentes idades e a necessidade de memória operacional fosse mais amplamente avaliada. Pelo fato do subteste Dígitos Ordem Direta utilizar com mais intensidade a memória do que Dígitos Ordem Inversa, foram desenvolvidas pontuações de processos distintos para essas duas tarefas. Tendo em vista o exposto, o presente estudo teve por objetivo analisar a sensibilidade do Índice de Memória Operacional do WISC-IV na identificação de dificuldades em crianças e adolescentes com o diagnóstico de Transtorno de Déficit de Atenção e Hiperatividade (TDAH).

\section{Método}

\section{Participantes}

Os participantes eram atendidos no Núcleo de Neurodesenvolvimento conveniado pelo Sistema Único de Saúde em parceria com a Universidade Federal de Pelotas. Todos tinham uma avaliação anterior da neuropediatra com o diagnóstico de Transtorno de Déficit de Atenção e Hiperatividade, baseado na entrevista clínica segundo o DSM-IV, na Escala SNAP-IV e na ficha de observação do professor.

A amostra foi de conveniência composta inicialmente por 50 sujeitos, entre 6 e 16 anos, matriculados na rede escolar pública e privada. Foram fatores de exclusão crianças e adolescentes que tinham iniciado o tratamento medicamentoso para TDAH para que a conduta estabilizada não interferisse na identificação dos sintomas (casos falso-negativos), assim como crianças com capacidade intelectual $\leq 70$. Dessa forma, foram excluídos do estudo 10 participantes.

Com base no critério de idade e no diagnóstico da psicóloga do local, listaram-se os pacientes que constituiriam a população-alvo. As pesquisadoras fizeram contato telefônico com as famílias e, na medida em que aceitaram participar do estudo, foram constituindo o grupo amostral, o qual foi captado por três meses período disponível para a coleta de dados.

Participaram da amostra 32 meninos e oito meninas. Os grupos etários de maior representatividade - 52,5\% e 40\% foram, respectivamente, dos 10 aos 13
$\operatorname{anos}(N=21)$ e dos 6 aos 9 anos $(N=16)$; quanto ao tipo de escola, $82,5 \%$ estavam no ensino público $(N=$ 33). O tempo médio de acompanhamento neurológico foi de 24 meses $(D P=22,14)$ e $47,5 \%$ dos pacientes $(N=19)$ nunca tinham usado medicamentos apesar do diagnóstico de TDAH.

\section{Instrumentos}

Foram utilizados os seguintes instrumentos:

Para investigar os sintomas de desatenção e hiperatividade/impulsividade, utilizou-se a escala SNAP-IV (Mattos, Serra-Pinheiro, Rohde, \& Pinto, 2006), cuja versão é resultante da tradução validada pelo GEDA - Grupo de Estudos do Déficit de Atenção da UFRJ e pelo Serviço de Psiquiatria da Infância e Adolescência da UFRGS. A escala foi elaborada a partir dos sintomas do Manual Diagnóstico e Estatístico - IV Edição (DSM-IV) da Associação Americana de Psiquiatria.

Para a estimativa da inteligência geral com a finalidade de identificar o critério de exclusão, foi utilizada uma forma reduzida do WISC-III (Della Veccia, 2011), formada pelos subtestes Vocabulário e Cubos, pois, na ocasião da coleta dos dados (primeiro semestre de 2013), o WISC-IV não havia sido publicado no Brasil. A estimativa do QI Geral foi calculada pela soma dos pontos brutos obtidos nos dois subtestes e convertida em pontos ponderados segundo a faixa etária dos indivíduos, os quais foram multiplicados pelo número de subtestes que compõem o QI Verbal e o QI Execução. A ponderação e a interpretação dos escores seguiram as normas do manual de aplicação e correção do teste WISC-III (Wechsler, 2002).

$\mathrm{Na}$ avaliação do Índice de Memória Operacional (IMO), utilizou-se o material cedido pela editora Casa do Psicólogo para a realização da pesquisa. O WISC-IV recomenda a aplicação do subteste Aritmética apenas quando um dos subtestes padrões (Dígitos e Sequência de Números e Letras) não puder ser administrado, entretanto aplicaram-se as três provas com o objetivo de avaliar o desempenho das crianças com TDAH em cada uma delas. A soma dos pontos brutos dos subtestes foi convertida em pontos ponderados (média $=10$; $D P= \pm 3$ ) e estes, no IMO (média $=100 ; \mathrm{DP}= \pm 15$ ); seguindo as normas e tabelas de conversão do manual de aplicação e correção do teste WISC-IV, também cedidas pela editora.

\section{Procedimentos}

Com o aceite do local onde os dados foram coletados e a aprovação do projeto pelo Comitê de Ética 
da Universidade Católica de Pelotas, a avaliação psicológica ocorreu em uma das salas de atendimento do local. A coleta de dados foi feita em dois momentos, com duração em torno de 60 minutos. No primeiro, os pais/responsáveis receberam as informações sobre a pesquisa e assinaram o Termo de Consentimento Livre e Esclarecido. Após, foi realizada a aplicação da escala SNAP - IV e o preenchimento dos dados demográficos. Ao finalizar, foi entregue um folheto com dicas sobre o manejo com os portadores de TDAH. No segundo momento, com a criança/adolescente foi realizada a aplicação dos subtestes Vocabulário e Cubos do WISC-III, seguidos dos subtestes que formam o IMO do WISC-IV. Após análise dos resultados, foi realizada uma entrevista de devolução com os pais/responsáveis e entregue ao serviço um perfil com o desempenho dos participantes nos subtestes das Escalas Wechsler.

\section{Análise de Dados}

Os dados foram digitados e analisados por meio do Statistical Package for the Social Sciences (SPSS). Foram utilizadas estatísticas univariadas (frequências e médias) e bivariada (Correlação de Pearson).

\section{Resultados}

Com relação ao sexo, $80 \%$ dos participantes eram meninos, com idade média de 10 anos $(D P=2,32)$. Os sintomas do TDAH foram identificados, segundo os pais/responsáveis, com a Escala SNAP - IV. Os resultados mostraram que $57,5 \%$ das crianças ou adolescentes $(N=23)$ foram identificados tanto com sintomas de desatenção, como de hiperatividade/impulsividade, sugerindo TDAH do tipo combinado; $27,5 \%(N=11)$ com sintomas de desatenção e 15\% $(N=6)$, apenas com sintomas de hiperatividade/impulsividade.
Tomando-se como indicador de dificuldades escolares a repetência $(37,5 \%$ da amostra) e a distorção idade e série (47,5\% da amostra), na Tabela 1, observase a frequência de crianças e adolescentes da amostra nessas variáveis, considerando as séries iniciais $\left(1^{\mathrm{a}}\right.$ ao $5^{\mathrm{a}}$ ano) e séries finais ( $6^{\mathrm{a}}$ ao $9^{\mathrm{a}}$ ano) do ensino fundamental. Apresentam-se também as frequências observadas nos dados oficiais divulgados pelo INEP - Instituto Nacional de Estudos e Pesquisas Educacionais Anísio Teixeira, no Censo Escolar 2016, para o estado do Rio Grande do Sul. Os dados sugerem que os portadores de TDAH apresentam maiores dificuldades escolares que os estudantes em geral.

O funcionamento intelectual geral (QI Total Estimado) dos participantes, avaliado pela forma reduzida do Teste WISC-III, variou entre QI 78 e QI 136, com o QI médio de 99,10 (DP $\pm 15,77)$, encontrando-se a maior frequência de participantes (40\%) com QI entre 90 a 109, classificado na tabela de Wechsler (2002) como dentro da média. A correlação entre o QI Total Estimado e o QI do Índice de Memória Operacional foi de $0,37(p=0,017)$. A Correlação de Pearson positiva sugere que ambas variam na mesma direção; por exemplo, maior capacidade cognitiva está associada à maior desempenho em memória operacional. O IMO também foi correlacionado com a idade dos participantes $(r=0,75 ; p<0,01)$, concluindo-se que à medida que aumenta a idade, aumenta o desempenho em IMO.

A capacidade de Memória Operacional (QI IMO) variou entre QI 62 e QI 106; a média foi de 83,63 (DP $\pm 11,34)$ e a moda 88 , ambas consideradas rebaixadas quando comparadas com o escore esperado para a população geral (QI Médio = 100). Quanto ao Índice de Memória Operacional, uma evidência de validade de critério pôde ser observada por meio da correlação entre a soma dos escores brutos dos três subtestes que

Tabela 1.

Percentagem de Crianças e Adolescentes com Repetência e Distorção Série/Idade

\begin{tabular}{lcccc}
\hline & \multicolumn{2}{c}{ Repetência } & \multicolumn{2}{c}{ Distorção idade e série } \\
\hline \multirow{2}{*}{ Ensino Fundamental } & Amostra & Estado do RS* & Amostra & $\begin{array}{c}\text { Estado do RS* } \\
\%\end{array}$ \\
\hline Séries iniciais & $\%(\mathrm{~N})$ & $\%$ & $70 \%$ & $14,1 \%$ \\
& $66,7 \%$ & & $(14)$ & $34,2 \%$ \\
Série Finais & $(10)$ & $14 \%$ & $30 \%$ & $(6)$ \\
& $33,3 \%$ & & $70 \%$ & \\
\hline
\end{tabular}

\footnotetext{
${ }^{*}$ Fonte: http://portal.inep.gov.br/web/guest/indicadores-educacionais
} 
o constituem no WISC-IV e a variável idade $(r=0,75 ; p$ $<0,01)$, evidenciando que quanto maior a idade, maior o escore bruto dos sujeitos.

Pôde-se observar, na Tabela 2, que os três subtestes que compõem o Índice de Memória Operacional (Dígitos, Aritmética e Sequência de Números e Letras) encontram-se rebaixados, comparando-se com a média esperada (média $\pm 10 ; D P \pm 3$ ). Em relação aos escores observados na amostra americana em crianças com TDAH, o subteste Dígitos mostrouse discrepante.

\section{Discussão}

A população-alvo, identificada em no núcleo de neurodesenvolvimento, era constituída de uma listagem de pacientes atendidos em um determinado período. Na listagem inicial, já constava maior representatividade de escolares do sexo masculino. Apesar da amostra deste estudo ser de conveniência, constituída de crianças cujos familiares aceitaram participar da pesquisa, observa-se uma frequência maior de meninos. Esse dado que vem ao encontro de diversos estudos, como Rohde e Halpern (2004); Fontana, Vasconcelos, Werner, Góes e Liberal (2007); Souza, Simão, Lima e Ciasca (2011); American Psychiatric Association (2014); Marcon et al. (2016), que apontam que o TDAH é mais frequente no sexo masculino do que no feminino na população em geral.

Segundo Graef e Vaz (2008), Marcon, Sardagna e Schussler (2016) e Effgem et al. (2017), o uso de escalas para pais e professores podem fornecer dados sistematizados, que permitem uma visão mais objetiva, com dados quantitativos dos sintomas, objetivando integrar esforços no sentido de tornar o diagnóstico o mais detalhado possível, resultando em uma intervenção mais precoce e efetiva. A Escala de Atenção SNAP - IV aplicada aos pais/responsáveis teve por objetivo identificar a percepção deles em relação aos sintomas dos filhos, em avaliação posterior ao diagnóstico realizado por ocasião do início do tratamento no ambulatório. Os dados confirmaram o diagnóstico neuropediátrico prévio, fornecendo evidências para caracterizar o grupo clínico em estudo, uma vez que todos os casos foram incluídos na desatenção, na hiperatividade ou em ambos. O resultado demonstra que não houve alteração na percepção dos pais/responsáveis, em relação aos sintomas do TDAH.

O sintoma mais prevalente identificado no estudo foi o do tipo combinado, o qual compreende tanto os sintomas de desatenção como os de hiperatividade. Os dados observados vão ao encontro da investigação realizada por Dorneles et al. (2014), que também verificaram a prevalência de $58,5 \%$ de crianças e adolescentes com predominância do tipo combinado em uma amostra de 270 sujeitos. Em seus estudos, o tipo hiperativo $(13,7 \%)$ é o que apresentou o menor índice de incidência, com resultados semelhantes ao do presente trabalho. Por outro lado, encontram-se resultados que identificaram a prevalência do tipo desatento como mais frequente entre as crianças e adolescentes com dificuldades escolares (Oliveira, Ragazzo, Barreto, \& Oliveira, 2016; Rohde, Dorneles, \& Costa, 2006).

A frequência observada de repetências e distorções sugere que o TDAH pode comprometer o rendimento escolar. Segundo Rohde e Mattos (2003), isso se dá pelo fato de que a atenção seletiva a estímulos relevantes é condição necessária das aprendizagens em geral e, em especial, as escolares. Assim, a criança acometida por esse transtorno apresenta dificuldades para sustentar a atenção durante um tempo mais prolongado, podendo

Tabela 2.

Escores Ponderados Médios por Subtestes do WISC-IV

\begin{tabular}{lcccc}
\hline \multicolumn{2}{c}{ Amostra } & & \multicolumn{2}{c}{$\begin{array}{c}\text { Amostra Americana com grupo } \\
\text { clínico TDAH - WISC-IV }\end{array}$} \\
\hline Subtestes & $\begin{array}{c}\text { Escores } \\
\text { ponderados }\end{array}$ & $\begin{array}{c}\text { Desvio } \\
\text { Padrão }\end{array}$ & $\begin{array}{c}\text { Escores } \\
\text { ponderados }\end{array}$ & $\begin{array}{c}\text { Desvio } \\
\text { Padrão }\end{array}$ \\
\hline Dígito & 5,88 & 2,45 & 9,6 & 3,0 \\
Aritmética & 7,08 & 2,67 & 8,7 & 3,3 \\
Sequência de Números e Letras & 8,46 & 1,80 & 9,3 & 3,5 \\
\hline
\end{tabular}

*Wechsler, 2013.

Psico-USF, Bragança Paulista, v. 24, n. 1, p. 109-117, jan./ mar. 2019 
ocasionar prejuízos escolares e, consequentemente, uma possível reprovação. Estudos de Barkley (2008) indicam que estudantes com TDAH correm mais risco de fracassar na escola do que crianças sem TDAH e com inteligência equivalente.

Para o diagnóstico diferencial do TDAH, é preciso descartar a presença de deficiência intelectual, visto que esse transtorno pode causar problemas de atenção, hiperatividade e impulsividade. Os participantes do presente estudo, na sua maioria, apresentaram um QI Total Estimado na média, com resultados similares aos encontrados na literatura com as Escalas Wechsler para escolares. O WISC-III (Wechsler, 2002) e o WISC-IV (Wechsler, 2013) foram aplicados em crianças com TDAH identificando todos os escores médios em QI próximos da média normativa.

Foi analisada a associação do escore total do IMO com a idade dos participantes. Segundo Pasquali (2010), a idade é um critério externo que pode ser usado como variável para validar testes de inteligência. A correlação positiva com o construto sugere evidência de validade.

Mayes e Calhoun (2006), em seus estudos sobre o WISC-IV, verificaram que toda a amostra de 118 crianças e adolescentes americanos diagnosticados com TDAH - avaliados com o teste, apresentaram o IMO como a pontuação de menor índice. Não era foco do presente estudo avaliar todos os índices fornecidos pelo WISC-IV, mas, em relação ao IMO, os sujeitos alcançaram desempenho médio inferior. Os dados também corroboram com os resultados obtidos por Messina e Tiedemann (2009) e Pereira et al., (2012), os quais identificaram déficit nas funções de memória e atenção, quando avaliaram funções executivas de crianças com TDAH.

Em contrapartida aos resultados encontrados na presente investigação, a pesquisa de padronização americana do WISC-IV identificou entre os indivíduos com TDAH $(N=89)$, escores em IMO dentro da média (QI IMO $=96,1)$. Cabe ressaltar que a amostra era constituída de crianças e adolescentes que, na sua maioria, usavam medicação (64\%) para o transtorno, fato que pode melhorar o desempenho no teste de inteligência (Wechsler, 2013).

Em relação aos resultados ponderados nos subtestes que formam o Índice de Memória Operacional, Wechsler (2013) identificou que Aritmética teve escores inferiores a Dígitos e Sequência de Números e Letras. Em contrapartida, no presente estudo, em Dígitos os participantes obtiveram o pior desempenho em relação aos demais subtestes.
Tomando-se os escores ponderados médios (10 \pm 3) dos subtestes, o escore diminuído em Dígitos sugere baixa capacidade de atenção, memória e concentração. Os resultados em Aritmética e Sequência de Números e Letras, apesar de estarem na média, sugerem um leve déficit nessas habilidades e os resultados são similares aos encontrados na amostra de padronização americana, com o grupo clínico de TDAH. Dessa forma, identifica-se que o subteste Dígitos parece a prova mais sensível para diagnosticar déficit de atenção e/ou hiperatividade, corroborando com os estudos de Guadagnini e Simão (2016).

Em relação ao subteste Sequência de Números e Letras, identificou-se uma contradição entre o rigor das regras de administração e a leniência da pontuação. Consigna: "- Agora eu vou dizer um grupo de números e letras. Quando eu terminar, quero que você repita primeiro os números, em ordem, começando pelo número mais baixo. Depois você diz as letras em ordem alfabética" (Wechsler, 2013, p. 125). Tal enunciado coloca o sujeito na situação em que precisa selecionar a informação relevante em cada problema, exigindo atenção sustentada e seletiva para o manejo da grande quantidade de informação que é gerada (identificar as letras, os números e colocar cada grupo em ordem crescente).

Apesar da exigência da consigna, para correção, o manual orienta que a resposta é pontuada como correta mesmo que a criança diga primeiro as letras e depois os números. Dessa forma, nas três tentativas do item 1, do item 2 e na primeira e segunda tentativa do item 3, se os números e letras usados como estímulos forem apenas repetidos pelo examinando como foram lidos pelo examinador, a criança automaticamente alcançará oito acertos, sem nenhum esforço das funções envolvidas pela prova. Esse escore bruto até a idade de 8 anos e 11 meses equivalerá a escores ponderados dentro da média (entre 7 e 10), segundo as Tabelas de Pontos Ponderados Equivalentes aos totais de pontos brutos nos subtestes, por grupo etário (Wechsler, 2013, p. 218 a 226).

Com base no exposto, Sequência de Números e Letras parece uma prova com pouco poder discriminativo da agilidade mental para as crianças menores de 9 anos, ao mesmo tempo alfabetizadas. Por outro lado, as crianças e adolescentes, independentemente da idade, que não completaram seu processo de alfabetização, terão dificuldades de realizar a tarefa. Nesse caso, como indica o manual (Wechsler, 2013), a prova deve ser substituída por Aritmética. 


\section{Considerações Finais}

O presente estudo teve por objetivo explorar algumas evidências da adequação do Índice de Memória Operacional na avaliação de crianças e adolescentes com TDAH. Os participantes foram captados em um núcleo de neurodesenvolvimento, com o diagnóstico prévio do transtorno, mas sem uso de medicação; eram na maioria meninos, um terço tinham histórico de repetência e apresentavam sintomas de TDAH do tipo combinado e do tipo desatento, segundo seus pais. Quanto à capacidade intelectual, a maior frequência dos participantes foi observada entre os QIs 90 a 109. Tais características são prevalentes nos grupos clínicos com TDAH.

O Índice de Memória Operacional envolve a habilidade de manter-se consciente de uma informação recebida, desenvolver uma atividade, saber manipulá-la e, a partir dela, produzir um resultado, envolvendo a atenção e a memória. Os portadores de TDAH geralmente têm alterações na memória e na atenção, as quais podem prejudicar seu desempenho escolar. $\mathrm{Na}$ avaliação do Índice de Memória Operacional no grupo clínico investigado, identificou-se um desempenho rebaixado, embasando a hipótese de que o IMO é sensível para identificar déficits nessas habilidades entre as crianças com TDAH.

Em relação aos subtestes que formam o Índice de Memória Operacional, identificou-se que Dígitos parece o mais indicado para avaliar os déficits de memória e de atenção, uma vez que nele observou-se a maior dificuldade de desempenho do grupo clínico. Questiona-se sobre a viabilidade do subteste Sequência de Números e Letras, manter-se como subteste padrão. A sugestão é torná-lo um subteste suplementar, considerando que apresenta algumas limitações para crianças e adolescentes com dificuldades de alfabetização, assim como leniência na pontuação.

Apesar de reconhecer a limitação do presente estudo, em relação à amostra de conveniência com número reduzido de participantes e com delineamento descritivo, os resultados apontam evidências que o Índice de Memória Operacional do WISC-IV é sensível para identificar dificuldades comuns entre crianças e adolescentes com TDAH. Com certeza, é um estudo exploratório, mas que colabora com evidências de validade do teste com grupos clínicos, uma vez que o o manual brasileiro do WISC-IV não apresenta estudos nesse sentido.
Seriam relevantes pesquisas em busca de evidências de validade baseadas nas relações com variáveis externas associando os escores do teste com outros instrumentos que meçam o mesmo construto ou construtos relacionados (validade convergente). Nesse sentido, os escores do WISC-IV poderiam ser correlacionados com escalas que avaliam o TDAH. Em busca de evidências de validade preditiva seriam importantes estudos com grupo clínico (portadores de TDAH) e grupo de controle (não portadores de TDAH).

\section{Referências}

Alves, G., Neme, C., \& Cardia, M. (2015). Avaliação neuropsicológica de crianças com transtorno do déficit de atenção e hiperatividade (TDAH) revisão de literatura. Revista Ibero-Americana de Estudos em Educação, 9(4), 760-769. Recuperado de https:// periodicos.fclar.unesp.br/iberoamericana/article/ view/7334

American Psychiatric Association. (2014). DSM V Manual diagnóstico e estatístico de transtornos mentais (5a ed.). Porto Alegre: Artmed.

Barkley, R. (2008). Transtorno de déficit de atenção/ hiperatividade ( $3^{\mathrm{a}}$ ed.). Porto Alegre, RS: Artmed.

Coutinho, G., Mattos, P., Schmitz, M., Fortes, D, \& Borges, M. (2009). Concordância entre relato de pais e professores para sintomas de TDAH: Resultados de uma amostra clínica brasileira. Archives of Clinical Psychiatry (São Paulo), 36(3), 97-100. doi: 10.1590/S0101-60832009000300003

Della Veccia, S. G. (2011). Formas Reduzidas do WISC-III: Características psicométricas de quatro modelos (Dissertação de mestrado não publicada). Programa de Pós-Graduação em Saúde e Comportamento da Universidade Católica de Pelotas, Pelotas, RS.

Dorneles, B. V. et al. (2014). Impacto do DSM-5 no diagnóstico de transtornos de aprendizagem em crianças e adolescentes com TDAH: Um estudo de prevalência. Psicologia: Reflexão e Crítica, 27(4), 759767. doi: org/10.1590/1678-7153.2014274167

Effgem, V., Canal, C. P. P., Missawa, D. D. A., \& Rossetti, C. B. (2017). A visão de profissionais de saúde acerca do TDAH - Processo diagnósticoepráticasdetratamento. Construçãopsicopedagógica, 25(26), 34-45. Recuperado de http://pepsic. 
bvsalud.org/scielo.php?script $=$ sci_arttext\&pi$\mathrm{d}=$ S1415-69542017000100005\&lng=pt\&tlng=pt

Fontana, R. S., Vasconcelos, M. M. de,Werner, J. Jr., Góes, F. V. de, \& Liberal, E. F. (2007). Prevalência de TDAH em quatro escolas públicas brasileiras. Arq. Neuro-Psiquiatr., São Paulo, 65(1). doi: 10.1590/S0004-282X2007000100027

Graeff, R. L., \& Vaz, C. E. (2008). Avaliação e diagnóstico do transtorno de déficit de atenção e hiperatividade (TDAH). Psicol. USP [online] 19(3), 341-361. doi: 10.1590/S0103-65642008000300005

Guadagnini, M., \& Simão, A. N. P. (2016). Investigação da atenção de adolescentes que apresentam mau desempenho escolar. Revista Psicopedagogia, 33(102), 251-261. Recuperado de ttp://pepsic.bvsalud.org/scielo.php?script=sci_ arttext\&pid $=$ S0103-84862016000300004\&lng $=p$ t\&tlng $=\mathrm{pt}$

Ignacio, M. G., Gonsalez, S. M. L., Almeida, C. C. R., Andrade, E. R de, \& Monteiro, E. R. (2008). Escala Wechsler de Inteligência para Crianças (WISC-III) na investigação do Transtorno do Déficit de Atenção/Hiperatividade (TDAH). Psicologia Hospitalar, 6(2), 61-73. Recuperado de http:/ / pepsic.bvsalud.org/scielo.php?script=sci_ arttext\&pid $=$ S1677-74092008000200005\&lng $=p t$ \&tlng $=\mathrm{pt}$

Lopes, R. M. F., Farina, M., Welter, G. W., Esteves, C. S., \& Argimon, I. I. L. (2012). Sensibilidad del WISC-III em la identificación del Trastorno de Déficit Atencional com Hiperactividad (TDAH). Cuadernos de Neuropsicología Panamerican Journal of Neuropshycho$\log$. 6(1), 128-140. doi: 10.7714/cnps/6.1.208

Marcon, G. T. G., Sardagna, H. V., \& Schussler, D. (2016). O questionário SNAP-IV como auxiliar psicopedagógico no diagnóstico preliminar do Transtorno de Déficit de Atenção e Hiperatividade (TDAH). Construção psicopedagógica, 24(25), 99-118. Recuperado de http://pepsic. bvsalud.org/scielo.php?script=sci_arttext\&pi$\mathrm{d}=$ S1415-69542016000100008\&lng=pt\&tlng=pt

Mattos, P., Serra-Pinheiro, M. A., Rohde, L. A., \& Pinto, D. (2006). Apresentação de uma versão em português para uso no Brasil do instrumento MTA-SNAP-IV de avaliação de sintomas de transtorno do déficit de atenção/hiperatividade e sintomas de transtorno desafiador e de oposição.
Rev. psiquiatr. Rio Gd. Sul, Porto Alegre, 28(3), 290297. doi: 10.1590/S0101-81082006000300008

Mayes, S. D., \& Calhoun, S. L. (2006). WISC-IV and WISC-III: Profiles in children with ADHD. Jounal of Attention Disorders. 9(3), 486-93. doi: $10.1177 / 1087054705283616$

Messina, L. F., \& Tiedemann, K. B. (2009).Avaliação da memória de trabalho em crianças com transtorno do déficit de atenção e hiperatividade. Psicol. USP. 20(2), 209-228. doi: 10.1590/ S0103-65642009000200005

Oliveira, D. B., Ragazzo, A. C. S. M., Barreto, N. M. P. V., \& Oliveira, I. R. (2016). Prevalência do transtorno de déficit de atenção e hiperatividade (TDAH) em uma Escola Pública da cidade de Salvador, BA. Revista de Ciências Médicas e Biológicas, 15(3), 354358. doi: 10.9771/cmbio.v15i3.18215

Pasquali, L. (2010). Instrumentação psicológica: Fundamentos e práticas. Porto Alegre: Artmed.

Pereira, A. P. P., Leon, C. B. R., Dias, N. M., \& Seabra A. G. (2012). Avaliação de crianças pré-escolares: Relação entre testes de funções executivas e indicadores de desatenção e hiperatividade. Rev. psicopedag. [online] 29(90), 279-289. Recuperado de http://pepsic. bvsalud.org/scielo.php?script=sci_arttext\&pi$\mathrm{d}=$ S0103-84862012000300002\&lng $=$ pt\&tlng $=$ pt

Rohde, L. A., \& Mattos, P., (2003). Princípios e práticas em $T D A H$. Porto Alegre: ArtMed.

Rohde, L. A., \& Halpern, R. (2004). Transtorno de déficit de atenção/hiperatividade: Atualização. J. Pediatr. (Rio J.), Porto Alegre, 80(2-supl). doi: 10.1590/S0021-75572004000300009

Rohde, L., Barbosa,G., Tramontina, S., \& Polanczyk, G. (2000). Transtorno de déficit de atenção/hiperatividade. Rev. Bras. Psiquiatr., 22(Suppl. 2), 07-11. São Paulo. doi: 10.1590/S1516-44462000000600003

Rohde, L. A., Dorneles, B. V., \& Costa, A.C. (2006). Intervenções escolares no transtorno de déficit de atenção/hiperatividade. Em Rotta, N. T. et al. Transtornos de Aprendizagem. Porto Alegre: Artmed (pp. 365-374).

Schwean, V. L., \& McCrimmon, A. (2008). WISC-IV Clinical Assessment and Intervention. San Antonio, Ed: Academic Press in an Imprint of Elsevier. 
Souza, G. G. B., Simão, A. N. P., Lima, R. F., \& Ciasca S. M. (2011). Desempenho cognitivo de crianças e jovens com Transtorno do Déficit de Atenção e Hiperatividade. Aletheia, (35-36), 69-78. Recuperado de http:// pepsic.bvsalud.org/scielo.php?script $=$ sci_ arttext\&pid=S1413-03942011000200006\&lng=pt $\& \mathrm{t} \operatorname{lng}=\mathrm{pt}$

Valença, A. M., \& Nardi, A. E. (2015) Histórico do diagnóstico do transtorno de déficit de atenção/ hiperatividade. Em A. E. Nardi, J. Quevedo, A. G. Silva (Eds.). Transtorno de déficit de atenção/hiperatividade: Teoria e Clínica. (pp.19-22). Porto Alegre: Artmed.

Vidal, F. A. S., Figueiredo, V. L. M., \& Nascimento, E. (2011). A quarta edição do WISC americano. Aval. Psicol., Itatiba, v. 10, n. 2, ago. Recuperado de http://pepsic.bvsalud.org/ scielo.php?script $=$ sci_arttext\&pid $=\mathrm{S} 1677$ $-04712011000200011 \& \operatorname{lng}=p t \& t \operatorname{lng}=$ $\mathrm{pt}$

Wechsler, D. (2002). WISC-III: Escala de Inteligência Wechsler para Crianças. Manual - $3^{\circ}$ ed. Adaptação e Padronização Brasileira: Figueiredo, V. L. M. São Paulo: Casa do Psicólogo.

Wechsler, D. (2013). Escala de Inteligência Wechsler para Crianças: WISC-IV. Manual de instruções para aplicação e avaliação. Adaptação e Padronização Brasileira: Rueda, F. J. M., Noronha, A. P. P., Sisto, F. F., Santos, A. A. A., \& Castro, N. R. C. 4ed. São Paulo: Casa do Psicólogo.

Recebido em: 13/06/2017 Reformulado em: 06/06/2018

Aprovado em: 30/07/2018

Sobre as autoras:

Carolina da Silva Büttow é psicóloga graduada pela Universidade Católica de Pelotas (UCPel), especialista em Psicopedagogia Clínica e Institucional pela Faculdade de Ciências Sociais de Florianópolis, FCSF/CESUSC, Brasil e atua como psicóloga clínica.

ORCID: 0000-0002-4089-7524.

E-mail: buttowcarol@gmail.com

Vera Lúcia Marques de Figueiredo é psicóloga graduada pela Universidade Católica de Pelotas (UCPel), pós-graduada no Mestrado em Psicologia da Pontifícia Universidade Católica do Rio Grande do Sul (PUC-RS) e no doutorado em Psicologia da Universidade de Brasilia (UnB) e atua como docente de graduação e de pós-graduação na UCPel desde 1980.

ORCID: 0000-0002-3580-0804.

E-mail:verafig@terra.com.br

Contato com as autoras:

Vera Lucia Marques de Figueiredo

Rua XV de Novembro, 457/apto 704

Centro- Pelotas-RS, Brasil

Tel.: (053) 3225-0273

Psico-USF, Bragança Paulista, v. 24, n. 1, p. 109-117, jan./ mar. 2019 